\title{
Gaugino Condensation in Heterotic Fivebrane Background
}

\author{
Noriaki Kitazawd* \\ Department of Physics, Tokyo Metropolitan University, Hachioji, Tokyo 192-0397, Japan
}

(Dated: December 24, 2018)

\begin{abstract}
The gaugino propagator is calculated by explicitly considering the propagation of a heterotic string between two different points in space-time using the non-trivial world-sheet conformal field theory for the fivebrane background. We find that there are no propagations of gaugino which is in the spinor representation of the non-trivial four-dimensional space of the fivebrane background. This result is consistent with the arguments on the fermion zero-modes of the fivebrane background in the low-energy heterotic supergravity theory. Furthermore, assuming the continuous limit to the flat space-time background at the place far away from the fivebrane, we suggest an effective propagator which is effective only at the place far away from the fivebrane in the flat space-time limit. From the effective propagator we evaluate a possible gaugino pair condensation. The result is consistent with the suggested scenario of the gaugino condensation in the fivebrane background in the low-energy heterotic supergravity theory.
\end{abstract}

*Electronic address: kitazawa@phys.metro-u.ac.jp 


\section{INTRODUCTION}

The quantum effect of gravity might be important in particle physics. For example, it was suggested that the supersymmetry can be spontaneously and dynamically broken by the gravitino pair condensation in non-trivial space-time backgrounds 1, 2]. It is interesting that this mechanism seems rather model independent in comparison with other mechanisms in field theory without gravity. Further investigation of this possibility requires a consistent theory of quantum gravity. Although string theory is a strong candidate of it, the dynamics of the fermion states in string theories has not yet been extensively studied. Especially, there are very few works about the fermion dynamics based on the framework of the string world-sheet theory. In this paper we discuss the dynamics of the gaugino in the fivebrane background of heterotic string theory using the technique of the world-sheet conformal field theory.

The fivebrane background is a non-trivial background of space-time metric and fields in heterotic and type II string theories [3, 4, 5]. The half of the original supersymmetry is broken, and the space-time Lorentz symmetry reduces to $\mathrm{SO}(5,1)$ from $\mathrm{SO}(9,1) \supset$ $\mathrm{SO}(5,1) \times \mathrm{SO}(4)$ by the fivebrane background. An interesting fact is that some special fivebrane backgrounds can be described by world-sheet conformal field theories as the solutions of string theories [6, 7]. We calculate the gaugino propagator in the fivebrane background of heterotic string theory perturbatively with respect to the string coupling without any low-energy approximations by using the world-sheet conformal field theory.

If we have a fermion propagator, or more precisely a fermion two point function, in some non-trivial backgrounds of the space-time metric and gauge fields, it may be possible to obtain a value of the fermion pair condensate which can be an order parameter of some symmetry breaking. For example, in Euclidean $\mathrm{SU}(N)$ gauge theory with one massless vector-like fermion in the fundamental representation of $\mathrm{SU}(N)$, the zero-instanton sector in the path integral of the fermion two-point function gives Euclidean propagator, and the one-instanton sector gives the value of the fermion pair condensate which triggers chiral symmetry breaking. Therefore, it is interesting to investigate the fermion two point function in non-trivial backgrounds.

The number of fermion zero-modes in the non-trivial four-dimensional space of fivebrane

backgrounds in low-energy supergravity theories is well-known [5] . In the fivebrane back- 
ground of heterotic string theory, there are two independent fermion zero-modes, and all three fermions, gaugino, gravitino and dilatino, have the zero-mode components[8]. If we believe the path integral formalism of the supergravity theory, this number means the gaugino pair condensation and no propagations of gaugino which is in the $\mathrm{SO}(4)$ (local Lorentz) spinor representation in the non-trivial four-dimensional space of the fivebrane background.

The paper is organized as follows. In the next section we give a brief review of the fivebrane background in the heterotic string theory. The world-sheet conformal field theory for the fivebrane background is introduced. In Sec III the gaugino propagator is calculated. The point boundary state which couple with a single gaugino state is introduced. The gaugino propagator is nothing but the transition amplitude between point boundary states of different places in space-time by the propagator operator in the world-sheet conformal field theory. The method of the calculation is the same in Ref. [9]. In Sec [V we discuss the limit of the fivebrane background to the flat space-time background at the place far away from the fivebrane. Based on the discussion, we suggest an effective gaugino propagator which continuously reduces to the ten-dimensional free propagator in the flat space-time limit. The effective propagator is effective only the place far away from the fivebrane. By using this effective propagator the gaugino pair condensation at the place far away from the fivebrane is calculated. In the last section we summarize our results.

\section{FIVEBRANE BACKGROUND IN HETEROTIC STRING THEORY}

The fivebrane background (or the NS5-brane) is a Bogomol'nyi-Prasad-Sommerfield (BPS) configuration which preserves half of the supersymmetry of the theory. In the heterotic supergravity theory, which is the low-energy effective theory of the heterotic string theory, the space-time metric and dilaton configuration of the fivebrane background (an NS5-brane at the origin) is explicitly given by

$$
\begin{gathered}
g_{a b}=e^{2 \Phi} \delta_{a b}, \quad g_{\bar{\mu} \bar{\nu}}=\eta_{\bar{\mu} \bar{\nu}}, \\
e^{2 \Phi}=e^{2 \Phi_{0}}+\frac{n \alpha^{\prime}}{r^{2}}
\end{gathered}
$$

where $a=1,2,3,4$ is the index of the non-trivial four-dimensional space, $r^{2}=x^{a} x^{a}$ is the

square of the distance from the fivebrane, and $n$ is some integer. We use $\mu=0,1, \cdots, 9$ as the index of the whole ten-dimensional space-time, and use $\bar{\mu}=0,1, \cdots, 5$ as the index 
of the flat six-dimensional Minkowski space-time in the whole ten-dimensional space-time. The geometry of the space-time is $M^{6} \times R \times S^{3}$ with varying radius of $S^{3}$ from $\sqrt{n \alpha^{\prime}}$ to infinity along with the value of the coordinate $r$ from 0 to $\infty$. The background configuration of the B-field strength and gauge field is

$$
\begin{array}{r}
H_{a b c}=-\epsilon_{a b c d} \partial^{d} \Phi, \\
A_{a}^{I}=2 \bar{\eta}_{a b}^{I} \partial^{b} \Phi,
\end{array}
$$

where $\bar{\eta}_{a b}^{I}$ is the 't Hooft $\eta$ symbol and $I$ is the index of the adjoint representation of SU(2) which is embedded in the original gauge group of the heterotic string theory $(\mathrm{SO}(32)$ in this paper). This solution in the low-energy supergravity theory is considered to be exact in the heterotic string theory. The world-sheet conformal field theory for these backgrounds can be explicitly constructed in case of $e^{2 \Phi_{0}}=0$. In this case the space-time geometry is $M^{6} \times W_{k}^{(4)}$, where $W_{k}^{(4)}$ is the four-dimensional group manifold of $\mathrm{SU}(2)_{k} \times \mathrm{U}(1)$ with Kač-Moody level $k$. The geometry of $W_{k}^{(4)}$ is again $R \times S^{3}$, but the radius of $S^{3}$ is fixed with $\sqrt{n \alpha^{\prime}}=\alpha^{\prime} / Q$, where $Q \equiv \sqrt{\alpha^{\prime} /(k+2)}$.

In case of the flat space-time the world-sheet theory of heterotic string theory consists of ten free boson fields $X^{\mu}(z, \bar{z})$, ten free fermion fields $\tilde{\psi}^{\mu}(\bar{z})$ and thirty-two free fermion fields $\lambda^{A}(z)$, where $A=1,2, \cdots, 32$. This system has $(N, \tilde{N})=(0,1)$ superconformal symmetry with the central charges of $c_{m}=10+36 / 2=26$ and $\tilde{c}_{m}=10+10 / 2=15$ which are cancelled by the ghost contributions of $c_{g}=-26$ (bc-ghost contribution) and $\tilde{c}_{g}=-26+11=-15$ (bc and $\beta \gamma$-ghost contributions). The non-trivial background $M^{6} \times W_{k}^{(4)}$ can be described by replacing unconstrained fields $X^{\mu}$ of $\mu=6,7,8,9$ by the fields constrained on the group manifold of $\mathrm{SU}(2)_{k} \times \mathrm{U}(1)$. Namely, the part of the world-sheet theory corresponding to the space-time coordinates of $\mu=6,7,8,9$ is replaced by the combination of the $\mathrm{SU}(2)_{k}$ WessZumino-Witten (WZW) model and the linear-dilaton theory. The new part has $(N, \tilde{N})=$ $(4,4)$ superconformal symmetry, and has the same central charge of the original part: $c_{m}^{4 D}=$ $\tilde{c}_{m}^{4 D}=6$, where we treat the fields $\lambda^{A}$ of $A=29,30,31,32$ as if they are superpartners of holomorphic boson fields.

The holomorphic sector of new $\mathrm{SU}(2)_{k} \times \mathrm{U}(1)$ part is described by three $\mathrm{SU}(2)_{k}$ bosonic currents $J_{i}(z)(i=1,2,3)$, one free bosonic current $J_{4}(z)=\partial X^{\mu=6}$ and four free fermionic fields $\Lambda_{a} \equiv \lambda^{28+a}$. These currents and fields satisfy the following operator product expansion. 
(We set $\alpha^{\prime}=2$ from now on in this section, for simplicity.)

$$
\begin{aligned}
J_{i}(z) J_{j}\left(z^{\prime}\right) & \sim-\frac{k}{2} \frac{\delta_{i j}}{\left(z-z^{\prime}\right)^{2}}+\epsilon_{i j l} \frac{J_{l}}{z-z^{\prime}}, \\
J_{4}(z) J_{4}\left(z^{\prime}\right) & \sim-\frac{1}{\left(z-z^{\prime}\right)^{2}}, \\
\Lambda_{a}(z) \Lambda_{b}\left(z^{\prime}\right) & \sim-\frac{\delta_{a b}}{z-z^{\prime}} .
\end{aligned}
$$

The $N=4$ superconformal symmetry transformation is generated by the following energymomentum tensor $T^{W_{k}^{(4)}}(z)$, supercurrents $G_{a}^{W_{k}^{(4)}}(z)$ and $\mathrm{SU}(2)_{n}$ currents $S_{i}(z)$.

$$
\begin{aligned}
T^{W_{k}^{(4)}} & =-\frac{1}{2}\left(\frac{2}{k+2} J_{i}^{2}+J_{4}^{2}-\Lambda_{a} \partial \Lambda_{a}+Q \partial J_{4}\right), \\
G_{i}^{W_{k}^{(4)}} & =\sqrt{\frac{2}{k+2}}\left(J_{i} \Lambda_{4}-\epsilon_{i j l} J_{j} \Lambda_{l}+\frac{1}{2} \epsilon_{i j l} \Lambda_{4} \Lambda_{j} \Lambda_{l}\right)-J_{4} \Lambda_{i}-Q \partial \Lambda_{i}, \\
G_{4}^{W_{k}^{(4)}} & =\sqrt{\frac{2}{k+2}}\left(J_{i} \Lambda_{i}+\frac{1}{3 !} \epsilon_{i j l} \Lambda_{i} \Lambda_{j} \Lambda_{l}\right)+J_{4} \Lambda_{4}+Q \partial \Lambda_{4}, \\
S_{i} & =\frac{1}{2}\left(\Lambda_{4} \Lambda_{i}+\frac{1}{2} \epsilon_{i j l} \Lambda_{j} \Lambda_{l}\right) .
\end{aligned}
$$

The background charge $Q$ determines the gradient of the linear-dilaton background $\Phi=$ $Q X^{\mu=6}$. The world-sheet field $X^{\mu=6}(z, \bar{z})$ is called the Feigin-Fuchs field, and the theory of this field is called the linear-dilaton theory. The value $Q=\sqrt{\alpha^{\prime} /(k+2)}=\sqrt{2 /(k+2)}$ is required to have the correct central charge of $c_{m}^{4 D}=6$, and the Kač-Moody level $n$ of SU $(2)_{n}$ is fixed to unity due to the relation of $n=c_{m}^{4 D} / 6$. The anti-holomorphic sector has exactly the same structure, except for that the fermion fields $\Lambda_{a}$ are replaced by $\tilde{\Psi}_{a} \equiv \tilde{\psi}^{5+a}$.

The world-sheet theory of the $M^{6}$ part consists of six free boson fields $X^{\bar{\mu}}(z, \bar{z})$, six free fermion fields $\tilde{\psi}^{\bar{\mu}}(\bar{z})$ and twenty-eight free fermion fields $\lambda^{\bar{A}}(z)$, where $\bar{A}=1,2, \cdots, 28$. In the holomorphic sector the energy-momentum tenor $T^{M^{6}}(z)$ is

$$
T^{M^{6}}=-\frac{1}{2} \partial X^{\bar{\mu}} \partial X_{\bar{\mu}}+\frac{1}{2} \lambda^{\bar{A}} \partial \lambda_{\bar{A}}
$$

In the anti-holomorphic sector the energy-momentum tenor and supercurrent are

$$
\begin{aligned}
\tilde{T}^{M^{6}} & =-\frac{1}{2} \bar{\partial} X^{\bar{\mu}} \bar{\partial} X_{\bar{\mu}}+\frac{1}{2} \tilde{\psi}^{\bar{\mu}} \bar{\partial} \tilde{\psi}_{\bar{\mu}}, \\
\tilde{G}^{M^{6}} & =\tilde{\psi}^{\bar{\mu}} \bar{\partial} X_{\bar{\mu}},
\end{aligned}
$$

respectively. The $M^{6}$ part has $(N, \tilde{N})=(0,1)$ superconformal symmetry. The whole worldsheet theory has $(N, \tilde{N})=(0,1)$ superconformal symmetry, and we take $T=T^{M^{6}}+T^{W_{k}^{(4)}}+$ 
$T^{g}, \tilde{T}=\tilde{T}^{M^{6}}+\tilde{T}_{k}^{W_{k}^{(4)}}+\tilde{T}^{g}$ and $\tilde{G}=\tilde{G}^{M^{6}}+\tilde{G}_{4}^{W_{k}^{(4)}}+\tilde{G}^{g}$ as the currents of that symmetry, where

$$
\begin{aligned}
& T^{g}=(\partial b) c-2 \partial(b c) \\
& \tilde{T}^{g}=(\bar{\partial} \tilde{b}) \tilde{c}-2 \bar{\partial}(\tilde{b} \tilde{c})+(\bar{\partial} \tilde{\beta}) \tilde{\gamma}-\frac{3}{2} \bar{\partial}(\tilde{\beta} \tilde{\gamma}), \\
& \tilde{G}^{g}=-\frac{1}{2}(\bar{\partial} \tilde{\beta}) \tilde{c}+\frac{3}{2} \bar{\partial}(\tilde{\beta} \tilde{c})-2 \tilde{b} \tilde{\gamma}
\end{aligned}
$$

are ghost contributions. The super-Virasoro generators $L_{n}, \tilde{L}_{n}(n \in \mathbf{Z})$ and $\tilde{G}_{r}(r \in \mathbf{Z}+1 / 2$ in Neveu-Schwarz sector and $r \in \mathbf{Z}$ in Ramond sector) are defined by

$$
\begin{aligned}
L_{n} & =\oint \frac{d z}{2 \pi i z} z^{n+2} T(z), \\
\tilde{L}_{n} & =-\oint \frac{d \bar{z}}{2 \pi i \bar{z}} \bar{z}^{n+2} \tilde{T}(\bar{z}), \\
\tilde{G}_{r} & =-\oint \frac{d \bar{z}}{2 \pi i \bar{z}} \bar{z}^{r+3 / 2} \tilde{G}(\bar{z}) .
\end{aligned}
$$

The explicit form of $L_{0}, \tilde{L}_{0}$ and $\tilde{G}_{0}$ for holomorphic Neveu-Schwarz and anti-holomorphic Ramond sector (NS-R sector) will be used in the next section.

The modular invariant partition function, or the one-loop vacuum amplitude, is explicitly given in Ref. [7] for even $k$. We consider $k$ as some even number from now on. One important fact is that in addition to the usual universal Gliozzi-Scherk-Olive (GSO) projection, the additional GSO projection is required for the modular invariance. The half breaking of the space-time supersymmetry by the fivebrane background is realized by the additional GSO projection in the world-sheet theory.

The additional GSO projection of the fivebrane background in $\mathrm{SO}(32)$ heterotic string theory also realizes the gauge symmetry breaking $\mathrm{SO}(32) \rightarrow \mathrm{SO}(28) \times \mathrm{SO}(4)$. The gauge boson state is

$$
\lambda_{-1 / 2}^{A} \lambda_{-1 / 2}^{B}|0\rangle_{N S} \otimes \tilde{\psi}_{-1 / 2}^{\mu}|0\rangle_{\widetilde{N S}},
$$

where $\lambda_{-1 / 2}^{A}$ and $\tilde{\psi}_{-1 / 2}^{\mu}$ are creation operators in NS and $\widetilde{\mathrm{NS}}$ sectors. In case that the worldsheet boson part of the state has equal $\mathrm{SU}(2)_{k}$ spins of the holomorphic and anti-holomorphic sectors, the additional GSO projection is nothing but the projection by the four-dimensional world-sheet fermion number. There are two four-dimensional world-sheet fermion numbers for each holomorphic and anti-holomorphic sector, $F$ and $\tilde{F}$, and $F+\tilde{F}$ should be even in the additional GSO projection. For even $\tilde{F}, \mu=0,1, \cdots, 5$ in Eq.(21), then $F$ should 
be even, and $A$ and $B$ should take $A, B=1,2, \cdots, 28$ or $A, B=29,30,31,32$ in Eq.(21), corresponding to the gauge bosons of $\mathrm{SO}(28)$ and $\mathrm{SO}(4)$ in six-dimensional space-time, respectively. In case of $\mu=6,7,8,9$, namely $\tilde{F}$ is odd, $F$ should be odd, and $A=1,2, \cdots, 28$ and $B=29,30,31,32$ in Eq.(21). These states correspond to four six-dimensional matter scalar fields in $(28,4)$ representation of $\mathrm{SO}(28) \times \mathrm{SO}(4)$.

The gaugino state is

$$
\lambda_{-1 / 2}^{A} \lambda_{-1 / 2}^{B}|0\rangle_{N S} \otimes\left|\mathbf{s}_{+}\right\rangle_{\widetilde{R}}
$$

where $\mathbf{s}_{+}$denotes the ten-dimensional spin state with even ten-dimensional chirality which is required by the universal GSO projection. In case of the four-dimensional chirality of $\mathbf{s}_{+}$is

even (six-dimensional chirality is also even), namely $\tilde{F}$ is even, $A$ and $B$ should take $A, B=$ $1,2, \cdots, 28$ or $A, B=29,30,31,32$ in Eq.(22) for even $F$. These states are corresponding to two six-dimensional gauginos of $\mathrm{SO}(28)$ and $\mathrm{SO}(4)$, respectively. In case of the fourdimensional chirality of $\mathbf{s}_{+}$is odd (six-dimensional chirality is also odd), namely $\tilde{F}$ is odd, $A=1,2, \cdots, 28$ and $B=29,30,31,32$ in Eq.(22) for odd $F$. These states are corresponding to two six-dimensional matter fermion fields in $(28,4)$ representation of $\mathrm{SO}(28) \times \mathrm{SO}(4)$. All these six-dimensional fermion states of Eq.(22) form six-dimensional $N=2$ supermultiplets with the boson states of Eq.(21). It is easy to check that other states, graviton, gravitino, dilaton and dilatino states, also form supermultiplets in six-dimensional $N=2$ supergravity theory [10].

\section{GAUGINO PROPAGATOR IN FIVEBRANE BACKGROUND}

The propagator in the heterotic string theory can be calculated as the transition amplitude between two appropriate boundary states by the propagator operator in the worldsheet conformal field theory [11, 12, 13]. This propagator can include some off-shell information [1]]. In order to obtain the propagator in the field theory limit (not the lowenergy limit), the point boundary state, which describes the state of the closed string shrinking to a point in space-time, should be used. We introduce the following point boundary state for the gaugino propagator in the fivebrane background.

$$
|B(y, \mathbf{s})\rangle^{A B}=\left|B_{X}^{M^{6}}\left(y^{\bar{\mu}}\right)\right\rangle \otimes\left|B_{\mathrm{FF}}\left(y^{6}\right)\right\rangle \otimes|C\rangle \otimes\left|\psi_{\mathbf{s}}\right\rangle^{A B}
$$


where each factor corresponds to the contribution of the bosons of $M^{6}$ part, the Feigin-Fucks field, the boson part of SU(2) $)_{k}$ WZW model and all the fermions, respectively. The explicit form of the first three factors is given in Ref.[9]. Here we just notice that $y^{\bar{\mu}}$ specifies a point in six-dimensional Minkowski space-time, $y^{6}$ specifies a point in the seventh space in $Q \rightarrow \infty$ limit and $|C\rangle$ describes the state of the closed string shrinking to the north pole of $S^{3}$ of radius $\alpha^{\prime} / Q$. The explicit form of the contribution of fermion fields is given by

$$
\begin{aligned}
\left|\psi_{\mathbf{s}}\right\rangle^{\bar{A} \bar{B}} & =\lambda_{-1 / 2}^{\bar{A}} \lambda_{-1 / 2}^{\bar{B}}|0\rangle_{\mathrm{NS}} \otimes|\mathrm{s}\rangle_{\tilde{\mathrm{R}}} \\
\left|\psi_{\mathbf{s}}\right\rangle^{\hat{A} \hat{B}} & =\lambda_{-1 / 2}^{\hat{A}} \lambda_{-1 / 2}^{\hat{B}}|0\rangle_{\mathrm{NS}} \otimes|\mathrm{s}\rangle_{\tilde{\mathrm{R}}}
\end{aligned}
$$

where $\bar{A}=1,2, \cdots, 28$ and $\hat{A}=29,30,31,32$, corresponding to $\mathrm{SO}(28)$ and $\mathrm{SO}(4)$ gauginos, respectively. Both ten and six dimensional chirality of these states are even.

The propagator is obtained by explicitly calculate

$$
P^{\left(A^{\prime} B^{\prime}\right)(A B)}\left(\psi_{\mathrm{s}^{\prime}}, \psi_{\mathrm{s}}, y^{\prime}-y\right)={ }^{A^{\prime} B^{\prime}}\left\langle B\left(y^{\prime}, \mathrm{s}^{\prime}\right)|D| B(y, \mathrm{~s})\right\rangle^{A B}
$$

where $A$ denotes $\bar{A}$ or $\hat{A}$ and $D$ is the propagator operator for NS- $\tilde{\mathrm{R}}$ sector:

$$
D=\frac{\alpha^{\prime}}{4 \pi} \frac{1}{2} \int_{|z| \leq 1} d^{2} z \frac{1}{|z|^{2}} \tilde{G}_{0} z^{L_{0}} \bar{z}^{\tilde{L}_{0}}
$$

The calculation is straightforward (see Ref. [9]) and the result is the following.

$$
\begin{aligned}
P^{\left(A^{\prime} B^{\prime}\right)(A B)} & \left(\psi_{\mathbf{s}^{\prime}}, \psi_{\mathbf{s}}, y^{\prime}-y\right)=-i \frac{\sqrt{\alpha^{\prime}}}{2} \frac{1}{\sqrt{2}}\left(\delta^{A^{\prime} A} \delta^{B^{\prime} B}-\delta^{A^{\prime} B} \delta^{B^{\prime} A}\right) \\
& \cdot \int \frac{d^{7} q}{(2 \pi)^{7}} e^{i q_{\tilde{\rho}}\left(y^{\prime}-y\right)^{\tilde{\rho}}} \bar{\psi}_{\mathbf{s}^{\prime}}\left(q_{\tilde{\sigma}} \Gamma^{\tilde{\sigma}}+\frac{Q}{\alpha^{\prime}} \frac{1}{3 !} i \epsilon_{i j k} \Gamma^{i} \Gamma^{j} \Gamma^{k}\right) \psi_{\mathbf{s}} \\
& \cdot \sum_{N_{X}=0}^{\infty} d\left(N_{X}\right) \sum_{l=0}^{k} \sum_{m=-\infty}^{\infty}(2(k+2) m+l+1) \sqrt{\frac{2}{k+2}} \sin \left(\pi \frac{l+1}{k+2}\right) \\
& \cdot \frac{1}{q^{\tilde{\lambda}} q_{\tilde{\lambda}}+\left(\frac{Q}{\alpha^{\prime}}\right)^{2}+M_{N_{X}}^{2}+\frac{4}{\alpha^{\prime}}\left((k+2) m^{2}+(l+1) m\right)},
\end{aligned}
$$

where $\Gamma^{\mu}$ is the ten-dimensional Gamma matrix, $\tilde{\rho}, \tilde{\sigma}, \tilde{\lambda}=0,1, \cdots, 6$ and $i, j, k=1,2,3$ with $\Gamma^{i} \equiv \Gamma^{6+i}$. The mass $M_{N_{X}}^{2}=4 N_{X} / \alpha^{\prime}$ depends on the bosonic level $N_{X}$ and $d\left(N_{X}\right)$ is the degeneracy of the open string state with the bosonic level $N_{X}$ and zero fermionic level. Since we are interested in the gaugino propagation, we set $N_{X}=0$ in the following. Furthermore, since the ten-dimensional space-time interpretation of this world-sheet theory is possible 
only in large $k$ limit, we take large $k$ limit. The result in the momentum space is very simple (the over all normalization is changed).

$$
P^{\left(A^{\prime} B^{\prime}\right)(A B)}\left(\psi_{\mathrm{s}^{\prime}}, \psi_{\mathrm{s}}, q\right)=\frac{1}{\sqrt{2}}\left(\delta^{A^{\prime} A} \delta^{B^{\prime} B}-\delta^{A^{\prime} B} \delta^{B^{\prime} A}\right) \bar{\psi}_{\mathrm{s}^{\prime}} \frac{q^{\hat{\mu}} \Gamma_{\hat{\mu}}+\frac{Q}{\alpha^{\prime}} \frac{1}{3 !} i \epsilon_{i j k} \Gamma^{i} \Gamma^{j} \Gamma^{k}}{q^{\hat{\mu}} q_{\hat{\mu}}+\left(Q / \alpha^{\prime}\right)^{2}} \psi_{\mathrm{s}} .
$$

We have no momenta in $S^{3}$ space, since we did not introduce a specific point in that space. We fixed the string at the north pole of $S^{3}$, but the absolute place of the north pole could not be fixed under the $\mathrm{SU}(2)_{k}$ symmetry. As a result, the propagation in $S^{3}$ is not incorporated 20]. The mass $\left(Q / \alpha^{\prime}\right)^{2}$ comes from the non-trivial torsion of the fivebrane background [14].

Now we consider the additional GSO projection. As discussed in the end of the previous section, the additional GSO projection is nothing but the six-dimensional chirality projection. The additional GSO projection requires to take $\psi_{\mathrm{s}}$ and $\psi_{\mathrm{s}^{\prime}}$ satisfying

$$
\frac{1+\gamma_{7}}{2} \psi_{\mathrm{s}}=\psi_{\mathrm{s}}, \quad \bar{\psi}_{\mathrm{s}^{\prime}} \frac{1-\gamma_{7}}{2}=\bar{\psi}_{\mathrm{s}^{\prime}}
$$

where $\gamma_{7} \equiv \Gamma^{0} \Gamma^{1} \cdots \Gamma^{5}$. Then the propagator becomes

$$
P^{\left(A^{\prime} B^{\prime}\right)(A B)}\left(\psi_{\mathbf{s}^{\prime}}, \psi_{\mathrm{s}}, q\right)=\frac{1}{\sqrt{2}}\left(\delta^{A^{\prime} A} \delta^{B^{\prime} B}-\delta^{A^{\prime} B} \delta^{B^{\prime} A}\right) \bar{\psi}_{\mathbf{s}^{\prime}} \frac{q^{\bar{\mu}} \Gamma_{\bar{\mu}}}{q^{\hat{\mu}} q_{\hat{\mu}}+\left(Q / \alpha^{\prime}\right)^{2}} \psi_{\mathrm{s}} .
$$

Note that there are no propagators of the gaugino in $\mathrm{SO}(4)$ (local Lorentz) spinor representations with $\Gamma^{\mu}$ of $\mu=6,7,8,9$ in the numerator. This is consistent with the arguments in low-energy effective theory. There are two $\mathrm{SO}(4)$ spinor zero-modes in non-trivial fourdimensional space in the fivebrane background in the low-energy supergravity theory[8], and there should be no $\mathrm{SO}(4)$ spinor propagations, if we believe the path integral formalism of supergravity theories. The instanton configuration in the non-trivial four dimensional space of the fivebrane background is not the one which indicates the instanton effect (the tunnelling effect between topologically non-trivial vacua), but the real configuration in fourdimensional Euclidean space. Therefore, the path integral in non-trivial four-dimensional space includes only "one instanton sector" without "zero instanton sector" and results no gaugino propagator.

We should keep in mind that the calculation in this section is in the lowest order of the string perturbation theory. Since the value of the string coupling $g_{s} \propto e^{\Phi}$ becomes large at the place of the fivebrane (see Eq.(2) with $e^{2 \Phi_{0}}=0$ ), the obtained propagator is effective only far away from the fivebrane (say $\left.r>\alpha^{\prime} / Q\right)$. 


\section{POSSIBLE GAUGINO CONDENSATION IN FIVEBRANE BACKGROUND}

Since the gaugino condensation is an off-shell effect, there are no standard techniques to calculate it in string theory. One of the possible strategy is to extract the information of the pair condensation from the propagator which includes some off-shell effect. This strategy is rather familiar in the field theory. For example, consider the quark propagator in QCD in four-dimensional space-time (though confinement might result no propagators of quarks). A typical form of the propagator is

$$
\text { F.T. }\langle 0|T q \bar{q}| 0\rangle=i \frac{p^{\mu} \gamma_{\mu}+\Sigma\left(p^{2}\right)}{p^{2}-\Sigma\left(p^{2}\right)^{2}}
$$

where F.T. means Fourier transformation and $\Sigma\left(p^{2}\right)$ is a function of $p^{2}$. Even though the quark is massless at the tree-level, the non-perturbative effect gives a non-trivial function of $\Sigma\left(p^{2}\right)$ and gives the constituent mass to the quark. The quark pair condensation can be roughly evaluated as

$$
\langle 0|\bar{q} q| 0\rangle \simeq-\operatorname{tr} \int \frac{d^{4} p}{(2 \pi)^{4}} \text { F.T. }\langle 0|T q \bar{q}| 0\rangle=-\int \frac{d^{4} p}{(2 \pi)^{4}} \frac{4 i \Sigma\left(p^{2}\right)}{p^{2}-\Sigma\left(p^{2}\right)^{2}} .
$$

The asymptotic freedom of the gauge interaction gives a finite value of the condensation. It is easy to see that the condensation like $\left\langle 0\left|\bar{q} \gamma^{\mu} q\right| 0\right\rangle$ vanishes.

If we naively apply this strategy to the gaugino propagator of Eq.(31), we have no condensations. This is consistent with the observation that there are no gaugino condensations in six-dimensions. The propagator can not be used to calculate the gaugino condensation in non-trivial four-dimensional space, since the method of the world-sheet conformal field theory with the additional GSO projection is not appropriate to describe the dynamics in non-trivial four-dimensional space. The string states which are selected by the additional GSO projection can be understood as the states in the supermultiplets in six-dimensional $N=2$ supergravity theory, but they can not be understood as the fields in four-dimensional space. This fact causes some difficulty to understand the physics in the flat space-time limit: $k \rightarrow \infty$ or $Q \rightarrow 0$. (Note that we have to go to infinitely far away from the place of the fivebrane to keep the perturbation on the string coupling effective. See Eq.(2) with $e^{2 \Phi_{0}}=0$ and the relation $\sqrt{n \alpha^{\prime}}=\alpha^{\prime} / Q$. This is the strong coupling limit in view of the whole theory.)

The flat space-time limit of the propagator of Eq.(31) does not coincide with the propagator in the flat ten-dimensional space-time. Because of the additional GSO projection, the 
half of the original states remain to be projected out. The same happens in the partition function in Ref.[7]. The partition function in the flat space-time limit is the half of the one in the flat space-time. These facts imply that the flat space-time limit do not give the heterotic string theory in flat space-time. This is due to the topological nature of the fivebrane background.

On the other hand, in the low-energy effective supergravity theory, all the non-trivial field configurations reduce to the trivial ones in the flat space-time limit at the place far away from the fivebrane. The local world-sheet dynamics also continuously reduces to the one in the flat space-time, since the world-sheet energy-momentum tensor and supercurrent continuously reduce to the one in the flat space-time. Therefore, it is natural to consider that the physics continuously reduces to the one in the flat space-time at least locally at the place far away from the fivebrane.

If this observation is correct, the effective propagator, which is effective only far away from the fivebrane, can be identified to the propagator of Eq.(29) without additional GSO projection. We can recover the spectrum in flat space-time by leaving off the additional GSO projection. The global property of the fivebrane background is neglected, and only the local world-sheet dynamics remains. Recovering the momentum in $S^{3}$ which now has very large radius,

$$
P_{\mathrm{eff}}^{\left(A^{\prime} B^{\prime}\right)(A B)}(q)=\frac{1}{\sqrt{2}}\left(\delta^{A^{\prime} A} \delta^{B^{\prime} B}-\delta^{A^{\prime} B} \delta^{B^{\prime} A}\right) \frac{q^{\mu} \Gamma_{\mu}+\frac{Q}{\alpha^{\prime}} \frac{1}{3 !} i \epsilon_{i j k} \Gamma^{i} \Gamma^{j} \Gamma^{k}}{q^{\mu} q_{\mu}+\left(Q / \alpha^{\prime}\right)^{2}} .
$$

This propagator continuously reduces to the one in flat space-time in $Q \rightarrow 0$ limit.

Now we can calculate the gaugino pair condensation. Following the strategy which has been explained in the beginning of this section, we have

$$
\left\langle\bar{\lambda}^{A B} \Gamma \lambda^{A B}\right\rangle \simeq-\int \frac{d^{10} q}{(2 \pi)^{10}} \operatorname{tr}\left(\Gamma P_{\mathrm{eff}}^{(A B)(A B)}(q)\right),
$$

where $\Gamma$ is some products of $\Gamma$-matrices. The calculation is straightforward, and we have only one possible gaugino condensation:

$$
\left\langle\bar{\lambda}^{A B} \Gamma^{7} \Gamma^{8} \Gamma^{9} \lambda^{A B}\right\rangle=-496 \times 32 \times \frac{\operatorname{Vol}\left(S^{9}\right)}{(2 \pi)^{10}} \cdot \frac{Q}{8 \alpha^{\prime 5}},
$$

where the first factor comes from the contraction of the gauge group indices, the second factor comes from the trace of the $\Gamma$-matrix, and $\operatorname{Vol}\left(S^{9}\right)$ is the volume of the nine-dimensional unit sphere. We have set the ultraviolet cut off $1 / \sqrt{\alpha^{\prime}}$ which is the natural cut off scale 
in the low-energy effective theory. This result is consistent with the scenario of the gaugino condensation which is suggested in Refs. [8, 15, 16]. The Lagrangian of the heterotic supergravity theory [17] contains a term

$$
\mathcal{L}_{\text {hetero }} \supset-\frac{1}{6 \kappa^{2}} e e^{-2 \Phi}\left(H_{\mu \nu \rho}-\frac{\kappa^{2}}{16} \bar{\lambda}^{A B} \Gamma_{\mu \nu \rho} \lambda^{A B}\right)^{2},
$$

where $\kappa \propto \alpha^{\prime 2}$ determines the gravitational scale in ten dimensions. In the fivebrane back-

ground $H$ has the non-trivial value of Eq.(3). Therefore, the composite operator $\bar{\lambda}^{A B} \Gamma_{\mu \nu \rho} \lambda^{A B}$ could be expected to condense for which the term of Eq.(37) vanishes for the vanishing cosmological constant. In the coordinate system of the present string world-sheet theory, only non-zero value of $H$ is $H_{789}=Q / \alpha^{\prime}$. Therefore, the condensation of Eq.(36) is totally consistent with this scenario.

This gaugino condensation may act an important role in the half supersymmetry breaking by the fivebrane background. In low-energy effective theory, we know the half supersymmetry breaking by the fact that the fivebrane background configuration is invariant under only the half of the original supersymmetry transformations. In the algebraic point of view, the partial supersymmetry breaking must be accompanied by the central charge in the supersymmetry algebra 18, 19]. It is well known that the central charge is induced by the topologically non-trivial configuration of the boson fields in space-time. There may be another contribution to the central charge through the quantum effect like the Konishi anomaly which gives a gaugino bilinear term in the supersymmetry transformation of some composite operators. There might be a possibility that the bilinear term of the gaugino (and also of the gravitino and dilatino) appears in the supersymmetry algebra in heterotic supergravity theory, and its condensation gives a contribution to the central charge for the half supersymmetry breaking by the fivebrane background (I would like to thank S.-J. Rey for pointing out this possibility).

\section{SUMMARY}

In string theory it is difficult to obtain the off-shell information, like the fermion pair condensation. The fermion propagator which may contain some information about the fermion pair condensation, can be concretely calculated by several methods in string theory, though no methods for the non-perturbative calculation are available. Since the fermion 
pair condensation is expected to be a non-perturbative effect, it seems impossible to treat it in string theories. But we know the instanton calculus in gauge theories in which the values of the condensates of fermion composite operators can be perturbatively calculated by the path integral in instanton backgrounds. The existence of the instanton configurations as classical solutions is itself has a non-perturbative meaning, and the non-perturbative effect is incorporated by the semi-classical approximation. It is expected that the similar situation is realized in the string propagator in non-trivial backgrounds.

Some non-trivial backgrounds in string theories are exactly described by using the worldsheet conformal field theory, and the propagators can be calculated perturbatively. The fivebrane background in the heterotic string theory is one of such background configurations, and the gaugino pair condensation in the non-trivial four-dimensional subspace of the space-time (and also the gravitino and dilatino pair condensations) is expected by the analysis in the low-energy supergravity theory. We calculated the gaugino propagator in the fivebrane background, and found no propagation of the gaugino in $\mathrm{SO}(4)$ (local Lorentz) spinor representation. Although this result is consistent with the arguments about the fermion zero-modes in the low-energy supergravity theory, we can not obtain the value of the gaugino condensate. The rigorous formulation for the fivebrane background allows to describe the dynamics only in six-dimensional space-time.

Based on the observation that in the flat space-time limit, $k \rightarrow \infty$, the physics at the place far away from the fivebrane should continuously reduce to the one in the flat spacetime, we suggested that the effective propagator at the place far away from the fivebrane is just the calculated propagator without the additional GSO projection. By using this effective propagator, we evaluated a possible gaugino pair condensate. The result is perfectly consistent with the suggested scenario of the gaugino condensation in low-energy effective supergravity theory.

The meaning and role of this condensation are unknown. One possibility is that the condensation contributes the central charge in the supersymmetry algebra in ten-dimensions. The central charge is required to realize the half supersymmetry breaking by the fivebrane. 


\section{Acknowledgments}

I would like to thank S. Saito for helpful comments. I also would like to thank S.V. Ketov for encouragements. This work was supported in part by Yamada Science Foundation (No. 2003-4022).

[1] E. Witten, Nucl. Phys. B188, 513 (1981); Commun. Math. Phys. 100, 197 (1985).

[2] K. Konishi, N. Magnoli and H. Panagopoulos, Nucl. Phys. B309, 201 (1988); B323, 441 (1989).

[3] S.-J. Rey, UCSB-TH-89/49, Invited talk given at Workshop on Superstrings and Particle Theory, Tuscaloosa, Alabama, Nov 8-11, 1989; Phys. Rev. D43, 526 (1991).

[4] C.G. Callan, J.A. Harvey and A. Strominger, Nucl. Phys. B359, 611 (1991).

[5] C.G. Callan, J.A. Harvey and A. Strominger, Nucl. Phys. B367, 60 (1991).

[6] S.-J. Rey, SLAC-PUB-5659, Presented at Particle and Fields '91 Conf., Vancouver, Canada, Aug 18-22, 1991.

[7] I. Antoniadis, S. Ferrara and C. Kounnas, Nucl. Phys. B421, 343 (1994).

[8] Y. Katagiri and N. Kitazawa, hep-th/0208069, to be published in Prog. Theor. Phys.

[9] N. Kitazawa, Phys. Rev. D68, 026005 (2003).

[10] H. Nishino and E. Sezgin, Nucl. Phys. B278, 353 (1986).

[11] A. Cohen, G. Moore, P. Nelson and J. Polchinski, Nucl. Phys. B267, 143 (1986).

[12] V.Ya. Fainberg and A.V. Marshakov, Phys. Lett. B211, 81 (1988).

[13] A.V. Marshakov, Nucl. Phys. B312, 178 (1989).

[14] E. Kiritsis and C. Kounnas, Nucl. Phys. B456, 699 (1995); Nucl. Phys. B422, 472 (1995).

[15] M.B. Green, J.H. Schwarz and E. Witten, "Superstring theory," Vol. 2, Cambridge Univ. Press.

[16] G.L. Cardoso, G. Curio, G. Dall'Agata and D. Lüst, hep-th/0310021

[17] E.A. Bergshoeff and M. de Roo, Nucl. Phys. B328, 439 (1989).

[18] E. Witten and D. Olive, Phys. Lett. B78, 97 (1978).

[19] B. Chibisov and M. A. Shifman, Phys. Rev. D56, 7990 (1997); ibid. D58, 109901 (1998).

[20] It is possible to incorporate the propagation in $S^{3}$ by fixing the string at the south pole in the final state. 